\title{
Die Pastoralinstruktion auf dem Hintergrund der päpstlichen Lehrtradition
}

\author{
von Giselbert Deussen
}

Die Pastoralinstruktion „Communio et progressio“ geht unmittelbar auf den Auftrag des II. Vatikanum zurück, dessen Dekret über die Mittel der sozialen Kommunikation "Inter mirifica“ in Nr. 23 den „ausdruicklichen Auftrag“ an den "Päpstlichen Rat für die Mittel der sozialen Kommunikation" erteilte, durch ein eigenes Pastoralschreiben „alle Grundsätze und Weisungen dieses Konzils über die sozialen Kommunikationsmittel zur Durchführung zu bringen"1. Auf diese Weisung bezieht sich die Instruktion, wenn sie im Vorwort betont, sie wolle "Lehrgrundsätze und pastorale Weisungen mehr im allgemeinen " darlegen. ${ }^{2}$ Damit stellt sie sich selbst in die kirchliche Lebrtradition, die aber weit über das Konzil hinausreicht. So verweist die Heraushebung von "Lehrgrundsätzen" („doctrinae principia“) auf einen Willen zur doktrinären Kontinuität, einer Fortsetzung der kirchlichen Lehrtradition, die in der katholischen Kirche mit der Ausübung der ordentlichen und außerordentlichen Lehrgewalt durch die Päpste deckungsgleich ist. Diese Kontinuität soll hier untersucht werden. Auf dem Hintergrund der päpstlichen Lehrtradition zu Fragen der Massenkommunikation wird sich die Pastoralinstruktion als ein ebenso brisantes wie typisches Exempel der Wandelbarkeit, Korrektionsbereitschaft und Widersprüchlichkeit päpstlicher "Lehrgrundsätze" erweisen.

Die Äußerungen des päpstlichen Lehramtes zu Fragen der Medienkommunikation, vornehmlich der Presse, sind zahlreicher und vielschichtiger, als man vermuten könnte. Deshalb können hier auch nur einige, allerdings beherrschende Aspekte herausgegriffen werden; zudem muß auch der Zeitraum der Untersuchung begrenzt werden. Wenn wir bei Gregor XVI. einsetzen, so ist damit nicht geleugnet, daß vorher schon eine lebhafte Auseinandersetzung der Päpste mit den neuen technischen Medien der Mitteilung stattgefunden hat, „wie das beispielsweise in den wiederholten Anweisungen und in der ausführlichen Begründung der kirchlichen Repressiv- und Präventivzensur zum Ausdruck kommt" 3 . Dennoch ist diese zeitliche Begrenzung nicht willkürlich: ist doch die Geburt einer eigentlich politischen Presse erst in das Frankreich der Jahre um die Revolution anzusetzen ${ }^{4}$, so daß auch in der kirchlichen Reaktion - einige Jahre später mit Gregor XVI. - eine neue Erörterungsphase dieser Phänomene einsetzt. Wenn wir ferner als beherrschende Aspekte 1. die ambivalente Überbewertung der "Großmacht Presse" und 2. die Lehrtradition zur Pressefreiheit abhandeln, so ist damit qualitativ wie quantitativ die Substanz der päpstlichen Stellungnahmen angegeben; alles andere ist Arabeske und Zugabe.

P. Giselbert Deussen, regelmäßiger Mitarbeiter von CS, wurde in diesem Jahr nach einem Ergänzungsstudium in Theologie, Sozialwissenschaften und Publizistik an der Universität Münster zum Dr. theol. promoviert; Thema seiner Dissertation: Ethik der Massenkommunikation bei Papst Paul VI. 


\section{Beberrschende Aspekte in päpstlichen Stellungnabmen zur Medienkommunikation}

von Gregor XVI. bis Pius XI.

\section{Die Übermacht der Medien}

Gregor XVI. und Pius IX., die sich dogmatisch und politisch zum schärfsten Konservatismus bekannten, sahen sich zu Beginn ihres Pontifikates heftigen Angriffen der „liberalistischen" Presse ausgesetzt. Gregor stand noch unter dem Schock der Revolution von 1830, die er im eigenen unbotmäßigen Kirchenstaat erlebte, als er der Presse pauschal vorwarf, daß sie nüberall die Fackeln heftigster Rebellion entzündet" habe, indem sie das Volk in seinen "Pflichten der Obrigkeit gegenüber wankend gemacht" habe. ${ }^{5}$ Pius IX. litt zeitlebens schwer unter dem Eindruck der römischen Revolution von 1848/49, die ihn ins Exil zwang und deren Ausbruch er der "gegnerischen Presse" zuschrieb. Diese Erfahrungen des Papsttums mit den Anfängen der politischen Presse im modernen Sinn ließen in die Lehräußerungen auch der Folgezeit ein Negativbild der Presse einfließen, das ihre Wirkungen bei weitem überschätzte und ihr eine Macht zuschrieb, die sie zu einem „Verführer des Menschengeschlechtes" hochstilisierte. Wie gebannt starte man angesichts der eigenen Ohnmacht auf die Möglichkeiten des bösen Feindes, dessen Erfolg man beneidete und den man für den eigenen Mißerfolg und das eigene Ungeschick im Umgang mit der modernen Offentlichkeit und deren Medien verantwortlich machte. Von dieser Mentalität her erklären sich die überspannten Kraftausdrücke und scharfen Verurteilungen in päpstlichen Verlautbarungen, wie folgende Liste von Klassifizierungen der als übermächtig empfundenen Presse zeigt:

Gregor XVI.: pestilenter Irrtum; Uberschwemmung mit Scheusalen von Irrlehren (1830-1846) und Wahnsinn; ${ }^{3}$ Ansteckung des Volkes durch die Ausschweifungen der Meinungs- und Pressefreiheit; Gefahr für Kirche und Staat; ${ }^{7}$

Pius IX.: lügnerischer, die Menschen betörender Verführer; ${ }^{8}$ Untergrabung (1846-1878) der Grundlage von Religion und bürgerlicher Gesellschaft; ${ }^{\theta}$ Verhetzung der Massen ${ }^{10}$

Leo XIII.: verderbliche Zügellosigkeit der "schlechten Presse “;11 gegen diese (1878-1900) hat die katholische Presse in "geschlossenen Reihen“" als "acies formata" $z u$ kämpfen;"12

Pius X.:

(1903-1914)

Pius XI.: absolute Zügellosigkeit; ${ }^{13}$

Kampf gegen die übermächtige „schlechte Presse“ und ihren unheilvollen Einfluß; ${ }^{14}$ liberalistische, laizistische Infektion. ${ }^{15}$

Während man die vermeintliche Übermacht der Medien und derer, die sie besitzen, beklagt und moralisch abqualifiziert, während diese zugleich eine Sündenbockfunktion erfüllen, betont man gleichwohl stets den Anspruch auf den Besitz dieser Mittel und ihre Kontrolle. Ein gebrochenes Verhältnis war die Folge: man möchte gerne die Mittel einsetzen, die man bei den anderen verabscheut, verketzert und verurteilt. Das magische Fascinosum des bösen Feindes wird deshalb "getauft ${ }^{\text {" }}$, indem man es als "Gesinnungsinstrument " eingemeindet, das erst in der Hand des glaubenstreuen, gehorsamen und in der Wahrheit stehenden Katholiken gute Früchte bringt. Dabei 
wird aber das Medium nicht ganz entzaubert: ein Rest von Mißtrauen gegen die Welt der Massenkommunikation bleibt stets zurück: Journalisten müssen immer besonders zu Gehorsam und Treue ermahnt werden, und ihrem Lebenswandel scheint man auch nicht so ganz zu trauen. Und wenn die "katholische Presse" so hoffnungslos wenig ausrichten kann, so ist doch wieder der übermächtige Sündenbock Presse daran schuld. Auch die katholische, die "gute" Presse bleibt mit dem magischen Odium des unheimlichen, weil so schlecht kontrollierbaren Beelzebub behaftet, durch den man gleichwohl den Teufel austreiben möchte.

\section{Die Lehrtradition zur Pressefreiheit}

Diese mehr gefühlsmäßige, stets gleichbleibende Attitüde gegenüber einer magisch empfundenen Ubermacht der Medien erhält ein rationales Komplement auf der Ebene der päpstlichen Staatslehre, in die die Lehre von der "wahren Preßfreiheit" einzuordnen ist. Diese Lehre hat eine interessante Entwicklung hinter sich. Nach undifferenzierten und kämpferischen Verdammungen der "verderblichen Preßfreiheit ${ }^{*}$ bei Gregor XVI. ${ }^{16}$ und Pius IX.17, die sich vor allem von dogmatischen Erwägungen über das „Recht der Wahrheit" leiten ließen, gegen das der einzelne, mündige Staatsbürger oder Gläubige keine Freiheitsrechte beanspruchen konnten, hat erst Leo XIII. eine systematische Lehre von der Freiheit der Publizistik vorgelegt. Obwohl wir auch bei ihm allgemeine Verurteilungen der Pressefreiheit finden, verwirft er dennoch nicht mehr die "Rede- und Pressefreiheit" schlechthin und in jedem Falle, sondern betont nunmehr, daß diese nur im Sinne einer "regellosen, alles Maß und alle Schranken überschreitenden Freiheit kein Recht auf Existenz besitzen kann "18.

Leo reduziert nämlich die Aufgabe der Presse im Staat 1. auf die Frage, ob die Freiheit der Meinung absolut sei oder nicht, und 2. auf das Verhältnis des Kommunikators zur Obrigkeit. Dabei denkt er deduktiv von einer hohen Ebene unvergänglicher Normen her, die in der Naturrechtsdoktrin begründet sind. Über dem Individuum steht die Norm des Ewigen Gesetzes Gottes, die er als Wahrheit bezeichnet, deren "Rechte höher stehen als die der Freiheit" ${ }^{\text {"19 }}$ und die aus der "Natur" abgelesen werden kann. Dieser Wahrheit sind auch die staatlichen Autoritäten verpflichtet, ${ }^{20}$ deren Herrschaftslegitimität sich unmittelbar aus dem Naturgesetz und damit von Gott herleitet. ${ }^{21}$ Die Herrschaft des Staates geht nicht vom Volke aus. ${ }^{22}$ Gegen theokratische Herrschaft, die in der Wahrheit der Gesetze Gottes sowohl erkannt wie auch normativ verankert ist, kann es keine autonomen, unbeschränkten Freiheiten geben, ansonsten könnte man sich auch gegen die Herrschaft Gottes auflehnen: „. . . es ist niemals erlaubt, die Gedankenfreiheit, Pressefreiheit und Lehrfreiheit . . zu fordern, zu verteidigen oder zu gewähren, als wären alle diese Freiheiten von Natur gegebene Rechte. Denn wären sie wirklich von Natur gegeben, dann wäre es Recht, Gottes Oberherrlichkeit zu bestreiten und kein Gesetz könnte der menschlichen Freiheit Grenzen setzen. "23

Die Korrespondenz des leonischen Wahrheits- und Herrschaftsbegriffs unterbindet also jede naturrechtliche Begründung der Meinungs- und Pressefreiheit, da sie dann nach Leos Prämissen auch gegen die Wahrheit und die von Gott verliehene weltliche Herrschaft durchgesetzt werden könnte, was aber in seinem System unmöglich, absurd ist. Die Wahrheit als höchste Norm politischer und gesellschaftlicher Prinzipien wollte und konnte Leo nicht dem freien Kräftespiel einer von Weltanschauungspluralismen geprägten demokratischen Gesellschaft überlassen: „Das bleibt jedoch immer wahr, 
daß eine allgemeine, unterschiedslos gewährte Freiheit . . an sich nicht begehrenswert ist; denn es widerspricht der Vernunft, daß der Irrtum gleiches Recht haben soll wie das Wahre."24 Damit ist einer Freiheit der Meinung als allgemeinem Menschenrecht, sowie dem Recht auf Information der Boden entzogen. ${ }^{25}$

Pius XI. greift die organische Staatsidee Leos auf, ordnet die Freiheit der absoluten Norm der Wahrheit unter und zwingt die Presse subordinatorisch in die Botmäßigkeit und respektvolle Gehorsamspflicht gegenüber der gottgewollten Obrigkeit, der allein das Recht der Kontrolle und Aufsicht gegen eine schrankenlose Freiheit der Offentlichen Meinung in der Presse zusteht. ${ }^{26}$ In dieser Offentlichkeit kommt dann der katholischen Presse, die als "gute Presse" einer andere als katholische Auffassungen vertretenden "schlechten Presse" gegenübergestellt wird, eine für ein demokratisches Gemeinwesen mit seinem notwendigen Meinungspluralismus unerträgliche Autorität zu: als gehorsames, willfähriges Instrument hoheitlicher Weisungsweitergabe steht sie eo ipso immer in der "Wahrheit", gegen die ja der "Irrtum“ kein Recht hat. Der heute noch oft zu beobachtende Medienklerikalismus und -triumphalismus kirchlicher Presseorgane dürfte hier seinen von primär dogmatischen Argumenten verursachten Entstehungsgrund haben. ${ }^{27}$

\section{I. Differenzierung der Lebre und neue Motive}

\section{Bei Pius XII. (1939-1959)}

Pius XII. betrachtet die demokratischen Systeme der Neuzeit mit Sympathie ${ }^{28}$, denn die Völker „fordern ein Regierungssystem, das mehr im Einklang steht mit der Würde und Freiheit der Bürger"29. Eine wirksame Garantie der Bürger- und Menschenrechte gegen eine das Individuum versklavende Staatsallmacht sieht er in der freien Meinungsäußerung und im guten Funktionieren der Offentlichen Meinung. Wo diese fehlt, wird nicht nur das Individuum gedemütigt und an seiner Entfaltung gehindert, sondern auch das ganze Sozialgefüge gestört: „Dort, wo überhaupt keine Äußerung der Offentlichen Meinung erscheint, vor allen Dingen dort, wo man ihr völliges Fehlen feststellen muß, wie immer ihr Schweigen oder ihr Fehlen sich erklären mag, muß man darin einen Mangel, eine Schwäche, eine Krankheit des gesellschaftlichen Lebens sehen. “30 Ein solchermaßen erkranktes Sozialgebilde führt zu „schändlicher Tyrannei" und ist "für die Journalisten und ihre Leser . . . eine demütigende Beleidigung " ${ }^{31}$. In der Offentlichen Meinung muß „die Mitgift jeder normalen Gesellschaft" gesehen werden, „sie ist letzten Endes überall das natürliche Echo, der mehr oder weniger ursprünglich gemeinsame Widerhall, den die Ereignisse und die augenblicklichen Verhältnisse in ihrem Geist und ihrer Urteilskraft finden" "32.

Pius XII. definiert also die Offentliche Meinung als Außerung des politischen Willens und Engagements des Bürgers und nicht nur als die des Publizisten. Jeder Bürger ist aufgerufen, am „Zwiegespräch der Gesellschaft ${ }^{\text {“33 }}$ teilzunehmen. Jedem Bürger muß die „rechtmäßige Freiheit" zugestanden werden, seine Stimme zu erheben: „Die Stimme der Bürger ersticken, sie auf ein erzwungenes Stillschweigen zurückzudrängen, bedeutet in den Augen aller Christen ein Attentat auf das natürliche Recht des Menschen, eine Verletzung der Weltordnung, wie sie Gott eingerichtet hat." 34 Hier wird ausdrücklich und zum ersten $\mathrm{Mal}$ - wenn auch in einer negativen Formulierung, die nicht den Normalfall des gesellschaftlichen Miteinanders, sondern den Ernstfall einer Bedrohung der persönlichen Freiheit beschwört - die Freiheit der Meinungsäußerung als Naturrecht bezeichnet. Wir finden jedoch bei genauer Durchsicht der 
zahlreichen publizistisch relevanten Außerungen Pius' XII. keinen eindeutigen Hinweis, der es erlaubt, diese naturrechtlich geforderte Meinungsfreibeit dergestalt auf die Freibeit der Presse und Information zu übertragen, daß behauptet werden könnte, Pius XII. habe auch die Pressefreiheit, d.h. die institutionalisierte Freiheit der Information und Meinung, als ein Naturrecht bezeichnet.

Eine Forderung des Naturrechts ist lediglich das Recht des einzelnen Bürgers auf freie Meinungsäußerung in der Offentlichkeit. ${ }^{35}$ Dem Journalisten kommt dabei eine Vermittlerfunktion zu, wobei auch er natürlich - qua Bürger - ein Recht auf freie Meinungsäußerung hat. Dieses Recht wird jedoch nie auf seine Funktion als Kommunikator oder auf die Funktion der Information im umfassenden Sinne bezogen. So werden auch, wenn der Papst von der Pressefreiheit spricht, der einzelne Journalist und die Presse als solche nicht als Rechtssubjekte, sondern als Verpflichtungssubjekte beschrieben.

Der Meinungsfreiheit als naturrechtlich begründbares individualethisches Menschenrecht entspricht also nicht auf der gleichen Ebene eine Freiheit der Presse als naturrechtlich zu fordernde sozialethische Norm. Die Pressefreiheit wird als "notwendig“ bezeichnet, nicht aber als Naturrecht. Vielmehr muß nach Pius im Zusammenhang mit dem "Schlagwort " Pressefreiheit eher von Verantwortung, Verpflichtung und Begrenzung gesprochen werden. ${ }^{36}$

\section{Bei Johannes XXIII. (1959-1963)}

Im Umfeld einer „Klimaänderung “ der päpstlichen Soziallehre, die sich nun grundsätzlich auf den Boden der Menschenrechte der Charta der Vereinten Nationen stellt, ${ }^{37}$ gelingt es Johannes XXIII., das Recht auf Information als ein Menschenrecht anzuerkennen, das auch naturrechtlich begründet ist: "Von Natur aus hat der Mensch ... das Recht, ... daß er frei nach der Wahrheit suchen und unter Wahrung der moralischen Ordnung und des Gemeinwohls seine Meinung äußern, verbreiten und jedweden Beruf ausüben darf; daß er schließlich der Wahrheit entsprechend über die öffentlichen Ereignisse in Kenntnis gesetzt wird. " ${ }^{38}$ Die oberste Sanktionsinstanz und die Garantie des „Funktionierens" von Wahrheit im individuellen und gesellschaftlichen Bereich sieht Johannes im Gegensatz zu seinen Vorgängern nicht in der staatlichen oder kirchlichen Autorität, sondern im freien, mündigen Gewissen des Menschen als einer allgemein gültigen Ordnungsnorm: "Jedoch hat der Schöpfer der Welt die Ordnung ins Innere des Menschen eingeprägt; sein Gewissen tut sie ihm kund und befiehlt ihm unbedingt, sie einzuhalten." ${ }^{\text {"3g }}$ Diese neuartige Verankerung des Rechtes auf freie Meinungsäußerung und Information und damit auch der Pressefreiheit im Naturrecht, wodurch die katholische Soziallehre den heimatlosen und lange von ihr geschmähten Gesellen moderner Menschenwürde in ihr Haus aufnimmt, zeigt die Wende, deren die päpstliche Soziallehre fähig ist. Die modernen Freiheiten, in "Mirari vos" noch als "verderbenbringend", „Wahnsinn" und "wider die Natur" verworfen, werden von Johannes als unveräußerliche Menschenrechte bezeichnet, die in Anspruch zu nehmen der Mensch verpflichtet ist. Nicht nur die neue geschichtliche Situation dürfte für diese Wende ausschlaggebend gewesen sein, sondern auch der systematische Ausgangspunkt dieses Papstes: nicht das geschichtslosabsolut Vorgegebene und das in die Unanfechtbarkeit und Endgültigkeit göttlicher Dekrete Hypostasierte, sondern das in die konkrete Geschichtlichkeit Hineingegebene und in die Anfechtung und Offenheit menschlich-fehlbarer Wahrheitssuche Preisgegebene definiert er als Basis und Agens menschlichen Zusammenlebens. Es geht nicht 
um die Durchsetzung absolut gesetzter "Wahrheiten", die die geschichtliche Weiterentwicklung nur allzu oft als Herrschaftsinstrument der Institutionsspitze zur Durchsetzung ihrer spezifischen Interessen entlarvt, sondern um das gesamtgesellschaftliche Funktionieren von zwischenmenschlicher Wahrhaftigkeit, in der Gottes Wahrheit geschichtlich erfahrbar wird. Garanten des Sieges dieser Wahrheit sind nicht die Autoritäten mit ihren Doktrinen und Dogmen, sondern die von Gott in jeden Menschen hineingelegte "Stimme des Gewissens“. Diese Stimme kann aber nur dort vernommen werden, wo die freie Suche nach der Wahrheit respektiert wird. Diese Suche darf nicht privilegierten Autoritäten vorbehalten sein, sondern muß im öffentlichen Gespräch aller Glieder der Gesellschaft in freier Kommunikations stattfinden. ${ }^{40}$

\section{Die Sonderstellung Pauls VI.}

Johannes verwendet zwar nicht den Begriff "Pressefreiheit", doch seine Auffassung von freier Meinungsäußerung und seine Definition des Rechtes auf wahrheitsgemäße Information setzen diese bei Berücksichtigung der heutigen Kommunikationsstrukturen voraus. Paul VI. blieb es vorbehalten, auf dem Boden des von Johannes eingeleiteten "Klimawandels" päpstlicher Sozialethik dieses in der Kirche lange verkannte, mißachtete und bekämpfte Recht nicht nur klar zu formulieren, sondern darüber hinaus eine differenzierte und wohl begründete Ethik der Pressefreiheit zu erarbeiten. ${ }^{41}$ "Informiert zu werden", ist nach Paul VI. deshalb in der Neuzeit „allgemein als ein universales, unverletzliches und unveräußerliches Recht des modernen Menschen anerkannt worden "42, weil seiner Ansicht nach 1. menschliches Zusammenleben heute ohne umfassende Information nicht mehr möglich ist ${ }^{43}$ und weil sie 2. einem Urbedürfnis der menschlichen Natur entspricht, weil Informationsrestriktionen die Personwürde des Menschen verletzen. ${ }^{44}$

Dieses Informationsrecht bezieht Paul VI. in einer für die päpstliche Soziallehre ganz neuen Variante des Beweises seiner Evidenz und Notwendigkeit auf das Funktionieren des menschlichen Zusammenlebens in den heutigen technisierten Massengesellschaften, so daß er zu sehr präzisen Beschreibungen der konkreten Gestalt dieses Rechtes kommt, wenn er z. B. die doppelte Dimension des Informationsrechtes als aktives Recht des Zugangs zur Information und als passives Recht auf Unterrichtung beschreibt ${ }^{45}$ : eine Eigenschaft, die die Publizistikwissenschaft die „Publizität einer Aussage" nennt und als die grundlegende Kategorie der Publizistik definiert, auf die alle weiteren Begriffe reduzierbar sein müssen. ${ }^{40}$ Aus der Publizität resultiert für ihn die Forderung nach der Freiheit der Information. Eine Begrenzung erfährt diese Freiheit nicht durch ein abstraktes und autoritäres Wahrheitspostulat, sondern nur durch eine sehr konkrete, auf die Person bezogene und ihre Sozialbindungen normierende humanitäre Kraft, die Paul VI. „Ehrfurcht“ nennt: Ehrfurcht vor den Ereignissen, d. h. Liebe zur Wahrheit und Hingabe an die Wirklichkeit, die er auch Objektivität nennt, ${ }^{47}$ und auch Ehrfurcht vor der Würde der Person und einer legitimen Intimsphäre. ${ }^{48}$ Der Fortschritt der päpstlichen Lehrtradition wird an dieser Konkretisierung einer etwaigen Begrenzung der Pressefreiheit besonders deutlich, wenn man die Auffassung Pauls VI. etwa mit dem abstrakten Axiom Leos XIII. vergleicht, das besagt, der Irrtum habe nicht das gleiche Recht wie die Wahrheit.49

\section{Die Pastoralinstruktion "Communio et progressio"}

Auf dem Hintergrund dieser Lehrtradition erscheint die Pastoralinstruktion weniger vom Himmel gefallen, als manche Presseberichte glauben machen. ${ }^{50}$ Motive konnten 
aufgegriffen, Ansätze weiterentwickelt und originäre, spezifische Eigenheiten katholischer Soziallehre eingebracht werden. Aber auch ganz wesentliche Korrekturen sind vorgenommen worden. Vor allem gegen die verhängnisvolle Tradition bis Pius XI. mußte sie sich ganz entschieden absetzen. So darf es als die eigentliche Errungenschaft des Schreibens, als eine Emanzipation aus einer Tradition des vor-rationalen Verhältnisses zur Publizistik, der autoritären Gängelei und renitenten Bestreitung der Freiheit der Information angesehen werden, wenn sie gegen eine unrealistische Definition der Massenmedien als machtvolle Instrumente zur kämpferischen Durchsetzung von Gesinnungen die Sachgerechtigkeit und gegen die Bestreitung der Pressefreiheit eine differenzierte ethische Begründung und Beurteilung der Kommunikationsfreiheit vorlegt.

\section{Der Primat der Sachgerechtigkeit}

Das Bemühen um sachlich fundierte Aussagen, von denen her dann erst Forderungen gestellt werden, scheint überall durch. Ob dies immer glücklich gelungen ist, muß einer ausführlicheren Kritik vorbehalten bleiben. Hier sei nur an einigen, allerdings zentralen Beispielen der Primat der Sachgerechtigkeit aufgezeigt, wie er vor allem im zweiten, kommunikationstheoretischen Teil zum Ausdruck kommt.

\section{a) Im Verständnis der Funktion der Offentlichen Meinung}

Die Instruktion schließt sich den Ergebnissen kommunikationswissenschaftlicher und soziologischer Forschungen sowie der Erfahrung in modernen Demokratien an, wenn sie die Offentliche Meinung und deren gutes Funktionieren als den wichtigen Sozialisationsfaktor bezeichnet. Auf dieser Erkenntnis beruht die Argumentation des ganzen Schreibens: „Die Außerungen und der Kampf ${ }^{[51]}$ der verschiedenen Meinungen in der Offentlichkeit greifen tief in das Leben der Gesellschaft ein, bereichern es und beschleunigen ihren Fortschritt. “ (Nr. 24). Konnte hier das Pastoralschreiben auch auf Pius XII., den es auch zitiert, zurückgreifen, so besteht der Fortschritt darin, daß die Rechte des einzelnen (des Kommunikators und Rezipienten) deutlicher betont werden, ohne die Sozialgebundenheit aller Mitteilungen zu übersehen, denn jeder soll bemüht sein, „seine eigenen Auffassungen, Meinungen, Empfindungen und sittlichen Überzeugungen anderen mitzuteilen, damit sie gemeinsamer Besitz vieler werden " (Nr. 25). Hört sich dieser Definitionsversuch des Forumcharakters moderner Offentlichkeit auch etwas allgemein und wenig präzise an, so fällt doch die Offenheit, Toleranz, die Orientierung auf die Essentials der Kommunikation in einer pluralistischen Gesellschaft auf, besonders wenn man sich an die Empfindlichkeit kirchlicher Funktionäre in Fragen der "sittlichen Überzeugungen “ erinnert: hier wird allen Gruppen der Gesellschaft zugestanden, ihre Uberzeugungen auf dem freien Markt der Meinungen zu handeln.

\section{b) Bedeutung und Funktion der Medienpädagogik}

Die Orientierung auf Sachzwänge und medienadäquates Verhalten drückt sich ferner im zweiten Kapitel über die „Voraussetzung für den sachgerechten Dienst der Instrumente der sozialen Kommunikation "52, insbesondere in den Abschnitten über die Notwendigkeit und Aufgabe einer Medienpädagogik aus: „Sie muß die Funktionsprinzipien der Instrumente der sozialen Kommunikation gründlich vermitteln." (Nr. 64). Diese Pädagogik muß sich sowohl auf die Rezipienten (Nr. 65-70) wie auf die Kommunikatoren (Nr. 71-72) beziehen. 
Medienpädagogik ist Sache von Familie und Schule, muß aber auch den Informator einbeziehen; als vordringliche Aufgaben zeichnet ihm das Pastoralschreiben vor: 1. eine sorgfältige Ergänzung seiner Informatorfunktion durch Kommentare, die von "Unparteilichkeit und ausgewogenem Urteil" (Nr. 76) zeugen und die den Zusammenhang der Informationen („das ganze Nachrichtengewirr") transparent machen und den Rezipienten in die Lage versetzen sollen, sich eine eigene Meinung in Konfrontation mit anderen Meinungen zu bilden (Nr. 74-77); 2. die Bedeutung einer gut funktionierenden Medienkritik, die darüber wachen soll, „daß das Angebot der Medien im Interesse des Publikums eine hohe formale und inhaltliche Qualität wahrt". Der Kritiker solle „eine Art beruflicher Selbstkontrolle“ bilden, „die es ersparen könnte, daß es zur Ausübung großen Drucks von außen kommt" (Nr. 78). Die gegenwärtige Diskussion um die innere Pressefreiheit zeigt die Aktualität dieses Vorschlags; 3. zur Sicherung und Institutionalisierung dieser Medienkritik wird eine Berufsorganisation „auf der Grundlage von Sachgerechtigkeit und Erfahrung " gefordert, „um gemeinsame Probleme und Aufgaben im Sinne der umfassenden Ziele sozialer Kommunikation zu lösen. Dabei wird es dienlich sein, Anleitungen und Anregungen zum rechten Handeln zu geben, statt einen Katalog von Verboten aufzustellen “ (Nr. 79); 4. die Verantwortung der Besitzer oder Verwalter von Medien (Nr. 80).

\section{c) Zusammenarbeit und Aufgabe des Staates}

Unter der Uberschrift "consociata opera" (Nr. 84-100) werden sowohl die Bürger wie auch die Träger staatlicher Gewalt an ihre Verantwortung für die gesellschaftliche Kommunikation erinnert. Zunächst wird der Gesetzgeber angesprochen, und zwar nicht - hier wird das Neue und die differentia specifica der Instruktion deutlich (s.u.) - als Kontrolle von Meinungsinhalten, sondern als Sicherung der Kommunikationsfreiheit; auch er darf seine Mitwirkung nicht in Einschränkungen und Verboten sehen, sondern er soll das Subsidiaritätsprinzip im Medienbereich zur Geltung bringen (Nr. 80). Eine Zensur darf nur das letzte Mittel sein und dann auch nur zum Schutze der Meinungsfreiheit (Nr. 87). Empfohlen werden gesellschaftliche Kontrollgremien, deren Besetzung die Pluralität der relevanten gesellschaftlichen Gruppen zum Ausdruck bringen sollte; diese Gremien seien am ehesten geeignet, "staatliche Eingriffe und ein Ubergewicht wirtschaftlicher Interessen" $\mathrm{zu}$ vermeiden (Nr. 88), sowie einen wirksamen Schutz der Jugend zu garantieren (Nr. 89).

\section{Das Recht auf Information und freie Meinungsäußerung}

Durchaus auf der Linie von Papst Paul VI. bezeichnet das Schreiben das Recht auf Information als freien Zugang zu den Quellen und Kanälen der Information, d. h. ais ungehinderte Unterrichtungsmöglichkeit, aber auch zugleich als Pflicht des Informationsgebers zu vollständiger, wahrheitsgemäßer Freigabe von Informationen. Auf Seiten des Rezipienten entspricht dem die Pflicht, sich auch tatsächlich zu informieren (Nr. 34). Neu und bemerkenswert ist dabei die Forderung nach Freiheit nicht nur des einzelnen Informators und einzelner Privatpersonen, sondern des gesamten Veröffentlichungsvorganges im Sinne einer institutionalisierten Informationsfreiheit, die den gesamten modernen, meist industrialisierten Informationsprozeß von der Recherche über die Veröffentlichung bis hin zum Empfang garantiert. Realistisch und berechtigt ist in diesem Zusammenhang die Kritik an den entstehenden Informations- 
und Meinungsmonopolen: „Ohne eine Vielzahl von einander unabhängiger Informationsquellen wäre der Gebrauch des Informationsrechtes völlig gegenstandslos" (Nr. 34); ferner die Forderung nach einem besonderen Rechtsschutz der Korrespondenten: „Die Menschen, deren Beruf es ist, die Offentlichkeit zu informieren, erfüllen daher eine sehr wichtige und schwierige Aufgabe, die sie oft großen Belastungen aussetzt. Häufig werden sie von denen unter Druck gesetzt, die ein Interesse daran haben, die Wahrheit zu verdunkeln und zu verschweigen." (Nr. 36.)

Eine weitere von der Lehrtradition abweichende, bzw. diese korrigierende Leistung besteht darin, daß ein uneingeschränktes Ja zur Meinungsfreiheit gesagt wird, die lediglich eine formale Begrenzung in ihrem Bezug auf das Gemeinwohl erhält. Weder staatliche noch kirchliche Autoritäten werden als Kontrolleure von Aussageinhalten angerufen; sie haben lediglich subsidiär die Freiheit des Aussageprozesses zu garantieren. Die Folgerungen daraus für den innerkirchlichen Kommunikationsprozeß müssen erst noch gezogen werden und dürften mit einer radikalen Veränderung der kirchlichen Kommunikationsstruktur gleichbedeutend sein, die bis in dogmatischjuridische Grundlagen des Kirchenbegriffes reichen.

\section{Kritische Anmerkungen}

Es dürfen aber auch Schwächen des Dokumentes nicht übersehen werden, die meist gerade auf der Affinität kirchlicher Aussagen, also auch dieses Schreibens, zur Tradition und somit auf die "Last des Uberkommenen " zurückzuführen sind. So wird z. B. manche entscheidende und fortschrittlich sich gebende Forderung an die allgemeine Offentlichkeit im innerkirchlichen Bereich nicht verifiziert. Das leidige Problem der kirchlichen Zensur wird ganz ausgespart. Bezüglich des Informationsrechtes in der Kirche bleibt der Text vieles schuldig, wenn man an die eklatanten Kommunikationsstörungen denkt, die für das gegenwärtige Kommunikationsbild der Kirche noch typisch sind. Hier zeigt sich eine Schwäche kirchlicher Dokumente, die darin besteht, der Gesamtgesellschaft mit recht vernünftigen und notwendigen Forderungen aufzuwarten, während man im eigenen Bereich nicht nur die Anwendung, sondern auch die grundsätzliche Revision von Strukturen mit dem Hinweis auf die „besondere Natur" der Institution Kirche schuldig bleibt. Etwas weniger Unbekümmertheit, etwas mehr Bescheidenheit und Bußfertigkeit und wenigstens das Durchscheinen eines entsprechenden Problembewußtseins hätten dem Text angestanden. Seine Verhaftung an tabuisierte Lehrgrundsätze scheint so denn auch an dem neuralgischen Punkt des kirchlichen Verhältnisses zur modernen Offentlichkeit durch, wenn es um die Zuordnung von Freiheit und Wahrheit geht. So spricht sie von „klaren Grenzen" des Rechtes auf Information, die aber in Wirklichkeit nie so klar gezogen werden können.

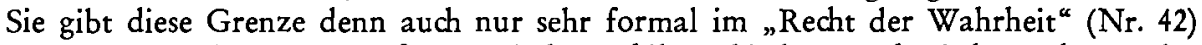
an. Dieses Axiom mag auf einer hohen philosophisch-metaphysischen Ebene seine Geltung haben. Aber auf die hier in Frage kommende äußere, politische Ordnung, insbesondere auf die pluralistische Gesellschaft mit ihren verschiedenen weltanschaulichen Wertorientierungen bezogen, kann nur der Mensch, und zwar auch der irrende, die Wahrheit verfehlende Mensch, Träger von Rechten sein und nicht die „Wahrheit". "Die Wahrheit hat Recht" würde in concreto doch immer nur heißen: diejenigen Menschen, die sich im Besitz der Wahrheit glauben, und die Instanzen, die über die Wahrheit entscheiden, haben Recht. ${ }^{53}$ Die hier (in Nr. 42) noch verschleierte Zuordnung von Wahrheit und Freiheit der Information wird schließlich offenbar und fatal, wenn in Bezug auf die innerkirchliche Kommunıkation bezeichnenderweise 
der freie Dialog nur auf den "Austausch legitimer Ansichten “ beschränkt bleibt (Nr. 116). Wer entscheidet denn in Staat und Kirche, wann Ansichten "legitim“ sind?

\section{Ein Kommentar als Traditionshüter}

Der von der deutschen Bischofskonferenz approbierten Ubersetzung ist ein langer Kommentar des Münchener Zeitungswissenschaftlers Hans Wagner beigegeben, der speziell auf die Lehrtradition eingeht (S. 86-98). Wagner kommt nun zu einer von unserer Darlegung erheblich abweichenden Interpretation der päpstlichen Lehre, die er durch die Brille seiner Lieblingsidee von dem sogenannten „Forumscharakter" der Medien und der "universalen Offentlichkeit" im Licht einer glorreichen Verteidigung der eigentlichen Kommunikationsfreiheit sieht: alle Verurteilungen der modernen Freiheiten von Gregor XVI. an werden da unter der Hand zu Zeugnissen eines mißverstandenen, aber der modernen Offentlichkeit zu Recht angesagten Kampfes gegen den "individualistischen Mißbrauch der Freiheit ${ }^{\text {" }}{ }^{54}$

Wagner übersieht dabei die Unter- bzw. Einordnung des päpstlichen Freiheitsbegriffes in das System der Korrespondenz von Wahrheit und Herrschaft, das wir darlegten. Ihm entgeht ferner, daß der Gemeinwohlbegriff vergangener Zeiten mit dem einer demokratischen Gesellschaft in vielen Punkten inkommensurabel ist. Auch die Offentlichkeitsstrukturen, die die Päpste im Blidk hatten, sind schlechthin unvergleichbar mit dem, was Wagner "universale Offentlichkeit" nennt. In der "Offentlichkeit", wie die päpstliche Lehrtradition sie verstand, war genau definiert, was Wahrheit ist, und genau bezeichnet, wer der Definitor und Organisator dieser Wahrheit war: die Obrigkeiten sakraler und säkularer Provenienz. Jeder Anspruch des Individuums mußte als ein Angriff auf diese Offentlichkeit empfunden werden, die keine Offentlichkeit für Untertanen, sondern für Standes-Inhaber und somit nicht universal, sondern in gewisser Weise totalitär war. ${ }^{55}$ Erst mit dem von Wagner notierten Auftreten ,individualistischer und subjektivistischer Offentlichkeitsansprüche ${ }^{\alpha}$ wurde die "totalitäre " Kommunikationsstruktur unterlaufen: Gerade dadurch fühlte sich die Kirche als Hüterin eines auf Herrschaft und Wahrheit metaphysisch-statisch fundierten Societas-Begriffs, der zudem noch von dogmatischen Attitüden geprägt war, aufs empfindlichste herausgefordert. Wagner sieht somit nur halb richtig, wenn er meint: "In Wirklichkeit deutete die Kirche konsequent die in der Neuzeit sich emanzipierenden Offentlichkeitsansprüche als Abfall von der Vollöffentlichkeit" ${ }^{\prime 56}$ - denn: "Abfall von der Vollöffentlichkeit" deutete diese Kirche nicht im Sinne des aufgeklärten Zeitgenossen Wagner ${ }^{57}$, sondern von der totalitären Offentlichkeit her, deren Repräsentanten die Hierokraten waren.

Anmerkungen:

1. Wir zitieren nach: Konzilsdekrete 4: Der Christ in der Welt, Redklinghausen 21966, S. 175 ; vgl. Nr. 19, die die Kompetenzzuweisung des päpstlichen Rates enthält (ebenda S. 174).

2. Nr. 3, zitiert nach: Päpstliche Kommission für die Instrumente der sozialen Kommunikation, Pastoralinstruktion Communio et Progressio über die Instrumente der sozialen Kommunikation, veröffentlicht im Auftrag des II. Vatikanischen Okumenischen Konzils (Nachkonziliare Dokumentation Band 11), Trier 1971. (Wir kürzen ab: CP); hier S. 153. 
3. A. Kollerbohm, Die ethischen Forderungen der katholischen Kirche an die Presse. Teildruck: Grundsätzliches um die Freiheit der Presse und Quellenkunde zu einer katholischen Presseethik, München 1936, S. 19.

4. Vgl. W. Bauer, Die öffentliche Meinung in der Weltgeschichte, Wildpark/Potsdam 1930, S. $239-257$.

5. Rundschreiben "Mirari vos ${ }^{\star}$ vom 15. 8. 1832, in: P. Dudon, Lamennais et le Saint Siège, Paris 1911, S. 397.

6. Ebenda S. 395 f.

7. Enzyklika "Singulari vos" vom 24. 6. 1834, in: Dudon S. 428.

8. Antrittsenzyklika vom 9. 11. 1846, in: Acta Pii IX Pontificis Maximi, Band I, Rom 1854, S. $25 \mathrm{ff}$.

9. Enzyklika „Quanta cura“ vom 8. 12. 1864, in: F. Rieß, Die Enzyklika Papst Pius' IX. vom 8. 12. 1864. Band I, Freiburg 1866, S. 2; vgl. Ansprache "Numquam fore" vom 15. 11. 1856, in: ders., Band XII, S. 56.

10. Konsistorialansprache vom 20. 4. 1849, in: Acta Pii S. $167 \mathrm{ff}$.; vgl. den "Syllabus“ im Anschluß an "Quanta cura" Nr. 79, in: E. Marmy (Hrsg.), Mensch und Gemeinschaft in christlicher Schau. Dokumente, Freiburg/Schweiz 1945, S. $53 \mathrm{f}$.

11. Ansprache "Ingenti sane laetitia“ vom 22. 2. 1879, in: S.D.N. Leonis P. XIII allocutiones, epistolae, constitutiones aliaque acta praecipua, Vol. I, Brügge 1887, S. 62-65; vgl. Enzyklika „Dall' alto" vom 15. 10.1890, in: Documenti pontifici sulla stampa (1878-1963), Rom/Vatican 1964 (Abkürzung: DPS), Doc. 7, S. 22. - Zum Begriff der "schlechten Presse“ vgl. M. Schmolke, Die schlechte Presse, Münster 1971.

12. "Sapientiae christianae" vom 10. 1. 1890, in: C. Ulitzka (Hrsg.), Leo XIII. der Lehrer der Welt, 1903, S. 192 f.; vgl. Enzyklika "Longinqua oceani“ vom 6. 1. 1895, in: C. M. Schneider, Die fundamentale Glaubenslehre der katholischen Kirche, vorgelegt und gegen die modernen sozialen Irrtümer verteidigt von Papst Leo XIII., Paderborn 1903, S. 165.

13. Vgl. Acta Pii X, Band I, Rom 1903, S. 54 f.

14. Vgl. Heilslehre der Kirche. Dokumente von Pius IX. bis Pius XII., hrsg. von P. Cattin/ H. Th. Conus, deutsch von A. Rohrbasser, Freiburg/Schweiz 1953, S. 1015, Nr. 1583; vgl. DPS, Nr. 214.

15. Vgl. DPS, Nr. 221.

16. "Mirari vos", in: Acta Gregorii XVI. Ausg. A. Bernasconi, Rom 1901, S. 169-174, und "Singulari vos", in: P. Dudon, a.a.O., besonders S. $428 \mathrm{f}$.

17. Vgl. "Quanta cura“ und Syllabus, a.a.O.

18. Enzyklika "Libertas praestantissimum“ vom 20.6. 1888, in: Rundschreiben Leos XIII. 3. Sammlung: De libertate humana, Freiburg 1888, S. 39. In den folgenden Ausführungen beziehen wir uns auf S. $49 \mathrm{ff}$.

19. Annum ingressi sumus ${ }^{\star}$ vom 19. 3. 1903, in: Ulitzka S. 13.

20. Vgl. "Libertas praestantissimum", in: Rundschreiben Leos XIII., S. 20 f.

21. Vgl. ebenda S. $16 \mathrm{f}$.

22. Vgl. "Annum ingressi“, in: Ulitzka S. 9; vgl. S. 153.

23. „Libertas", in: Rundschreiben Leos XIII., S. 56.

24. Ebenda S. 50.

25. Zur näheren, differenzierteren Argumentation bezüglich der Ablehnung einer scbrankenlosen Freiheit vgl. G. Deussen, Ethik der Massenkommunikation bei Papst Paul VI. (Dissertationsschrift) Münster 1971 , S. 55 f.

26. Quellen siehe ebenda S. $69 \mathrm{ff}$.

27. Diese Haltungen gingen z. B. in das Konzilsdekret "Inter mirificac ein, das den katholischen Publizisten aufgrund ihrer Unterordnung unter die kirchliche Autorität eine nahezu unfehlbare Kompetenz vor allem in Fragen der öffentlichen Sittlichkeit zuschreibt.

28. Vg1. A.-F. Utz/ J.-F. Groner, Aufbau und Entfaltung des gesellschaftlichen Lebens. Soziale Summe Pius' XII. 3 Bände, Freiburg 1954 ff. (Abkürzung: UG), Nr. 3501.

29. UG 3469.

30. UG 2134.

31. UG 2136.

32. UG 2133.

33. Pius XII. verwendet anstelle dieses Begriffes der funktionalen Publizistik den Ausdruck "das natürliche Echo", mit dem die gleiche Funktion der Offentlichen Meinung in einer demokratischen Gesellschaft beschrieben werden soll. Vgl. W. Hamerski, Reden Papst Pius’ XII. zu Fragen der Publizistik, in: „Publizistik“, Bremen, 8:1964, S. 615.

34. UG 2135. 
35. Vgl. UG 3474: „Seine eigene Meinung aussprechen zu können über die ihm auferlegten Pflichten und Opfer, nicht gezwungen zu sein zum Gehorchen, ohne erst gehört zu werden, das sind zwei Rechte des Staatsbürgers, die in der Demokratie, wie schon ihr Name sagt, ihren Ausdruck finden."

36. Vgl. UG $2101 \mathrm{ff}$.

37. Vgl. "Pacem in terris ${ }^{\star}$ Nr. 143, in: A.-F. Utz (Hrsg.), Die Friedensenzyklika Papst Johannes' XXIII. (Herder-Bücherei Band 157), Freiburg 1963, S. 130.

38. Ebenda Nr. 12.

39. Ebenda Nr. 5.

40. Wegen der gebotenen stofflichen Begrenzung bleibt hier die interessante Verbindung der Aufgabe der Kommunikationsmittel mit dem Freizeitproblem, welches ein besonderes Anliegen Johannes' XXIII. war, außer Betracht.

41. Vgl. G. Deussen, Ethik und Massenkommunikation bei Papst Paul VI., in: „Communicatio Socialis" $2: 1969$, S. 306 ff.

42. Audienzansprache vom 17. 4. 1964, in: Insegnamenti di Paolo VI (Tipografia Poliglotta Vaticana) $1965 \mathrm{ff} ., 6$ Bände (Abkürzung: INS), hier: Band II, S. 253-255.

43. Vgl. u.a. Brief an R. Manzini vom 30.6. 1968, in: „Acta Apostolicae Sedis“, Vatican 1968, LX, S. $471 \mathrm{f}$.

44. Vgl. z. B. INS II, S. 253 f.

45. INS II, S. 253.

46. Vgl. F. Dröge, Publizistik und Vorurteil, Münster 1967, S. 90.

47. Vgl. u.a. Rede vor Journalisten vom 23. 8. 1968, in: INS VI, S. 381 f,; ferner: INS VI, S. $210-213$; INS II, S. $137-141$.

48. Vgl. INS II, S. 253-255; INS III, S. 247-250, S. $536 \mathrm{f}$.

49. Vgl. "Libertas praestantissimum“, in: Rundschreiben Leos XIII., a.a.O., S. 50.

50. Vgl. z. B. A. Schlitter, Nach dem Reformwerk gab es Wermut, in: „Frankfurter Rundschau" vom 10. 6. 1971, S. 8.

51. Die lateinische Fassung spricht hier richtiger von "certatio“ (Wettstreit), (CP n. 24, S. 174).

52. Präzise formuliert die lateinische Uberschrift: „Condiciones aptissimae ad actionem congruentem" (CP, S. 204).

53. Vgl. E.-W. Böckenförde, Religionsfreiheit als Aufgabe des Christen, in: „Stimmen der Zeit“" 170. Band, 90:1964/65, S. 206.

54. Vgl. CP, S. 89. Wagner steht damit in der Tradition von A. Kollerbohms Studie: Die ethischen Forderungen der katholischen Kirche an die Presse, a.a.O., der auch an entscheidender Stelle von Wagner (S. 92) zitiert wird. Für Kollerbohm singen alle päpstlichen Außerungen das Hohelied der "wahren" und "wirklichen" Pressefreiheit, das dann aber bezeichnenderweise stets in den Kehrvers von "ethischen Forderungen der katholischen Kirche an die Presse" einmündet; Kollerbohms Freiheitsbegriff ermöglicht eine Interpretation der Quellen, die die Päpste immer und in allem zu Anwälten der ${ }_{m}$ wahren Freiheit" deklariert; diese "Freiheit" ist eine zweifache: einmal Freiheit der katholischen Kirche von staatlicher Bevormundung (Kollerbohm S. 95 f.), zum anderen - Freiheit, bzw. Säuberung der Offentlichkeit von sitten- und wahrheitswidrigen Meinungen und Gesinnungen durch eben diese Autoritäten ( $S .17 \mathrm{ff}$.).

55. Vgl. G. Deussen, Verkündigungsstrategien im historischen Rückblick, in: „Communicatio Socialis" $4: 1971$, S. $1-10$.

56. CP, S. 93 .

57. Gleichwohl würde der Demokratiebegriff Wagners auch in diesem Zusammenhang interessieren, wenn er dem liberalistischen Denkmodell den „uneingeschränkten Optimismus“ vorwirft,, $\mathrm{da} B$ in einem geheimnisvollen Selbstregulierungsprozeß sich die Wahrheit schon an den Tag fördern, das Zusammenleben der von Natur aus guten Menschen schon erreichen lasse, wenn nur eine durch keine Autorität oder Macht eingeschränkte individuelle Freiheit durchgesetzt werden könne ${ }^{a}$ (S. 93). Welch anderer Optimismus bleibt denn einem aufrechten Demokraten als die skepsis-kontrollierte Hoffnung, daß diese "Schwäche ${ }^{*}$ des demokratischen Systems: das Gute und Wahre gegen den Willen seiner Bürger nicht erzwingen zu können und ihrer Vernunft und Mündigkeit ausgeliefert zu sein, letztlich doch besser ist als alles Mißtrauen, das die Vorstufe zu Reglement, Unfreiheit und endlich Terror ist? 


\section{S U M M A R Y}

The modern popes' instructions from Gregory XVI to Pius XI on mass media (in the concrete: on the first mass media, the press) were influenced by the distrust in the "basic rights" of the French Revolution - especially in the freedom of speech and freedom of the press. Pius XII changed the instruction by showing the "public opinion" as necessary function of the human society. By this he gave to the individual the right of contributing to the formation of the public opinion. John XXIII has liberated mankind with regard to the modern liberties and basic rights from the subjection to the formal authorities of state and Church, and he has taken the conscience as a norm. Paul VI especially pointed out the "claim on information". Against the badkground of this tradition the pastoral instruction of "Communio et Progressio" is to be regarded. It took up all the positive developments of instruction and enriched them by the knowledge of the science of communication. It ignores some commitments to ancient tradition of teaching. The comment of the German edition (author: Hans Wagner) is more traditional than the instruction itself.

\section{RESUMEN}

Las instrucciones de los Papas de Gregorio XVI hasta Pío XI sobre los medios de masas (concretamente: sobre el primer medio de masas, la Prensa) estaban bajo la influencia de la disconfianza a los „derechos señoriales “de la Revolución Francesa - especialmente a la libertad de manifestar una opinión y la libertad de la Prensa. Pío XII ha cambiado la instrucción, presentando la „opinión pública “ como función necesaria de la sociedad humana. Por eso atribuía al individuo el derecho de contribuir a la formación de la opinión pública. Juan XXIII ha liberado al hombre de la sumisión a las autoridades formales del estado y de la Iglesia y presentaba la voz interior como norma. Paulo VI ha especialmente acentuado el "derecho a información". Con el fondo de esta tradición la instrucción pastoral de „Communio et Progressio“ debe ser visto. Se ha valida de todos los desarrollos positivos de la instrucción y los ha enriquecidos con el saber de la ciencia de comunicación. Ignora algunas relaciones con la antigua tradición de instrucción. El comentario de la edición alemana (autor: Hans Wagner) muestra más tradicionalismo que la instrucción misma. 